\title{
CLINICAL TRIALS: PERSPECTIVES OF MEDICAL COMMUNITY
}

\author{
HARI SANKAR KN, DHANYA SASIDHARAN PALAPPALLIL, PRABITHA PANATTIL
}

Department of Pharmacology, Government Medical College, Kottayam, Kerala, India. Email: drspdhanya@gmail.com

\author{
Received: 09 May 2020, Revised and Accepted: 12 June 2020
}

\begin{abstract}
Objective: The objective of the study was to determine the knowledge, attitude, and perception about clinical trials (CTs) among medical community of a tertiary care institution.

Methodology: This was a questionnaire-based cross-sectional study conducted in the Department of Pharmacology, Government Medical College in Central Kerala for a period of 1 year. In a sample population of 690 comprising medical undergraduates ( ${ }^{\text {rd }}$ phase), residents, and doctors in Medical Education Service (MES), 309 questionnaires were distributed randomly assuming 80\% response to achieve the sample size of 247 . Knowledge, attitude, and perceptions were quantified with a cumulative score with a scoring scheme of +1 for a correct response/positive attitude/positive perception and 0 for an incorrect response/no response/negative attitude/negative perception. The data were analyzed using SPSS 16, the knowledge, attitude, and perception were expressed as mean score as well as proportion.
\end{abstract}

Results: The response rate was $95.79 \%$. Of the 296 completed responses, 158 (53.4\%) were from medical undergraduates, 62 (20.9\%) from residents, and $76(25.7 \%$ ) from doctors in MES. The mean knowledge was $14.8 \pm 3.79$ (maximum score 25). The mean attitudinal score was $7.07 \pm 1.82$ (maximum score 10). The mean score of the participants on perception (maximum score $=10$ ) about CTs conducted in India was $4.11 \pm 2.02$ which denoted a negative perception.

Conclusion: The participants had good knowledge and positive attitude about CTs, however, all the three groups of participants had negative perception about CTs currently conducted in India.

Keywords: Clinical trial, Knowledge, Attitude, Perception, Questionnaire.

(C) 2020 The Authors. Published by Innovare Academic Sciences Pvt Ltd. This is an open access article under the CC BY license (http://creativecommons. org/licenses/by/4. 0/) DOI: http://dx.doi.org/10.22159/ajpcr.2020.v13i9.38228

\section{INTRODUCTION}

Clinical trials (CTs) of drugs are prospective ethically designed investigation in human subjects to objectively discover, verify, or compare the results of two or more therapeutic measures [1]. They are designed to acquire information about the pharmacokinetic and pharmacodynamic properties of a candidate drug [2]. Information from CTs help patients, clinicians, policy-makers, and funding agencies to make clinical and health policy decisions $[3,4]$.

The ultimate objective of CT is to emanate the benefits of intervention to patients and community at large. Unfortunately, some of the practicing physicians are unaware of the actual procedures involved and the real purpose of CTs which lead on to a negative perception towards it. Nevertheless, CTs are conducted only when there is enough evidence of expected positive benefit-risk ratio, but sometimes disappointing outcomes can occur which are often misinterpreted by both media and public [5]. Infamous scandals and the constant reports of fraudulence by some of the pharmaceutical companies have grimaced the integrity and ethics in CT among both public and clinicians. The "guinea pig" status of the participants and inevitable role of the pharmaceutical industry gives an exploitative image to investigators and sponsors [6]. In addition, the protocol design complexity implemented in the past two decades has increased both the cost and duration of CTs. All these encourage opposition to CTs and seed distrust among clinicians which can be deleterious to the indigenous scientific scenario of the country [7].

By its quality of being focused terrain, the science of CT may not be well understood by those who are not specifically trained in it. A lack of knowledge may transmute into an adverse discernment toward CT. It is essential for every clinician to update his knowledge/skills to give the best available treatment. Evidence-based medicine plays a significant role in medical practice and its advancement. Hence, every clinician should cultivate adequate knowledge and develop right attitude for clinical research and CTs. The medical students in medical colleges are the cornerstone of next-generation medical fraternity. Hence, they also should have a basic idea about CTs to improve the existing medical education system and thus to foster research culture [8]. However, data regarding the knowledge and perception of CTs among doctors and medical students are scanty, what available promotes the need for conduct of more such studies. Accordingly, this questionnairebased study was conducted to determine the knowledge, attitude, and perception about CTs among medical community which comprised medical undergraduates, residents, and doctors in Medical Education Service (MES) of a tertiary care institution.

\section{METHODOLOGY}

This was a questionnaire-based cross-sectional study conducted in the Department of Pharmacology, Government Medical College in Central Kerala from December 1, 2018, to November 30, 2019, for a period of 1 year. Medical undergraduates in the third-phase MBBS ( $\mathrm{n}=280)$, residents $(n=130)$, and doctors in MES $(n=280)$ made up a total sample population of 690 . From the total population, a sample size of 247 was computed at $95 \%$ confidence level allowing $5 \%$ margin of error [9]. A list of the sample population was created and they were assigned a consecutive number from 1 to 690 . Simple random sampling was done from the random number table for each stratum. A total of 309 questionnaires were distributed assuming $80 \%$ response.

Questionnaire was prepared based on literature search on similar survey and standard CT guidelines $[5,10,11]$. The construct validity was done by formal opinion on each item by subject experts $(n=3)$. The 
questionnaire was piloted among postgraduates from same setting in the department of pharmacology $(n=6)$. The questionnaire thus prepared had items on three domains - knowledge (25 questions), attitude about CTs (10 questions), and perception about CTs in India (10 questions). Knowledge, attitude, and perceptions were quantified with a cumulative score with a scoring scheme of +1 for a correct response/positive attitude/positive perception and 0 for an incorrect response/no response/negative attitude/negative perception. Before conducting the analysis, the internal consistency of instrument was assessed for reliability and Cronbach's coefficient alpha 0.64. Clearance from the Institutional Research Board was obtained before the study.

Written informed consent was obtained from the participants before filling the print questionnaire. In the Google fill up Form, those not consenting could skip the questionnaire and directly submit it. All participants who gave informed consent were included in the study. The investigators distributed the questionnaire to participants either as print or emails according to the convenience of the participants. At least two reminders were sent by message/email to return the completed questionnaire. The data were entered into Excel sheet and analyzed using SPSS 16 for Windows (SPSS Inc., Chicago, USA). The knowledge, attitude, and perception were expressed as mean score as well as proportion.

\section{RESULTS}

Of the 309 questionnaires distributed, 300 were returned, out of which four were eliminated because of incomplete response, thus making a response rate of $95.79 \%$. The mean age of the participants was 27.49 \pm 9.01 years with 192 (64.9\%) females and 104 (35.1\%) males. Of the participants, 158 (53.4\%) were medical undergraduates, $62(20.9 \%)$ were residents, and $76(25.7 \%)$ were doctors in MES. The mean knowledge was $14.8 \pm 3.79$ (maximum possible score was 25 ). Table 1 shows that only $30 \%$ of participants had read articles or books on CT, $15.5 \%$ knew that both CT Registry of India (CTRI), and Institutional Ethics Committee should approve a CT and 33.8\% did not know about the possibility of receiving monetary incentives for the participants. Only $30.7 \%$ knew about the post-trial access benefits and only 33\% knew about the role of CTRI in disseminating CT-related information to the public.
The mean attitudinal score was $7.07 \pm 1.82$ (maximum score 10 ). As shown in Fig. 1, majority of the participants had a positive attitude toward CTs. Attitude was considered positive when participant responded positively for positive statements and negatively to negative statements. Majority of the participants disagreed to the statement that CTs are unethical, incorrect, and purely inhuman $(224,75.67 \%)$, conducting CTs are waste of time, workforce, and money $(273,92.2 \%)$, and compensations for study-related injury needs not be reimbursed to the patients $(226$, $76.35 \%$ ). However, majority of the participants thought that CTs were conducted because of insufficient research data $(203,68.5 \%)$ and majority was unwilling to participate as a subject in CTs $(199,67.23 \%)$.

The mean score of the participants on perception (maximum score=10) about CTs conducted in India was $4.11 \pm 2.02$ which denotes a negative perception. Fig. 2 shows the disagreement to various statements on CTs in India. As shown in Fig. 2, majority of the participants inclusive of residents and doctors in MES thought that informed consent processes of CT done in India were not really informed, patients were deprived of the standard treatment in Phase 3, patients got influenced by the high monetary incentives.

\section{DISCUSSION}

Nowadays, medical education and clinical practice are more evidence based for which CTs lay the foundation. The information disseminated through research and CTs are utilized for prevention, prophylaxis, diagnosis, and cure/palliation of diseases. Many published research trials were instrumental in bringing forth major changes in medical education and practice. CTs help clinicians to choose the best therapy for each patient [12]. Hence, good knowledge, positive attitude, and perception about CTs among medical fraternity are very essential and possibly it is presently one of the key challenges in the medical research field. This study was done to evaluate the extent of knowledge and assess the attitude and perception of the medical students, residents, and faculty about CTs in a tertiary care center.

The mean knowledge was 14.8 \pm 3.79 (maximum possible score was 25). A vast majority (93.6\%) knew that informed consent was essential for CT. Although the participants have a fairly good knowledge in general, the knowledge about preclinical animal studies, informed consent, continuation of standard treatment after trial period, patients right

Table 1: Responses which scored +1 in the knowledge domain

\begin{tabular}{|c|c|c|}
\hline Knowledge domain & 1 & $\mathbf{0}$ \\
\hline 1. Have you read any articles or books on CT & $90(30.4)$ & $206(69.6)$ \\
\hline 2. CT protocol should be approved by CTRI not IEC & $46(15.5)$ & $250(84.5)$ \\
\hline 3. Informed consent is mandatory for CT & $277(93.6)$ & $19(6.4)$ \\
\hline 4. Placebo is a dummy drug & $250(84.5)$ & $46(15.5)$ \\
\hline 5. Standard treatment is always discontinued in CT & $182(61.5)$ & $114(38.5)$ \\
\hline 6. Patients are not supposed to receive monetary incentives for CT & $100(33.8)$ & $196(66.2)$ \\
\hline 7. A patient once enrolled in a trial cannot withdraw till study completion & $217(73.3)$ & $79(26.7)$ \\
\hline 9. CT for a new drug can be done without animal toxicity study reports & $221(74.7)$ & $75(2)$ \\
\hline 10. Patients identity is usually not disclosed to sponsor & $254(85.8)$ & $42(14.2)$ \\
\hline 11. After completion of CT, patients receive free medication & $91(30.7)$ & $205(69.3)$ \\
\hline 12. Drug-free washout is for complete elimination of drug from body & $188(63.5)$ & $108(36.5)$ \\
\hline 13. The head of drug regulatory authority in India DCGI & $175(59.1)$ & $121(40.9)$ \\
\hline 14. Good Clinical Practice is the day-to-day guideline for clinical practice & $200(67.6)$ & $96(32.4)$ \\
\hline 15. Principal investigator may be blacklisted if there is malpractice & $237(80.1)$ & 59 (19.9) \\
\hline 16. CTRI system disseminate CT-related information to public & $99(33.4)$ & $197(66.6)$ \\
\hline 18. Study drug-related adverse effects are reported as adverse events in CT & $223(75.3)$ & $73(24.7)$ \\
\hline 19. It is unethical to conduct CT in healthy volunteers & $220(74.3)$ & $76(25.7)$ \\
\hline 20. CTs cannot be sponsored by government funding agencies & $182(61.5)$ & $114(38.5)$ \\
\hline 21. DCGI approves manufacture and import of new drugs & $250(80.4)$ & $46(19.6)$ \\
\hline 22. Informed consent includes benefits and not the potential true risks & $134(45.3)$ & $162(54.7)$ \\
\hline 23. There is no need to conduct CT for a new use of drug & $263(88.9)$ & $33(11.1)$ \\
\hline 24. Most Phase III CTs are randomized, double-blind, controlled trials & $191(64.5)$ & $105(35.5)$ \\
\hline 25. Post-marketing surveillance of drug detects how fast the drug is sold & $97(32.8)$ & $199(67.2)$ \\
\hline
\end{tabular}




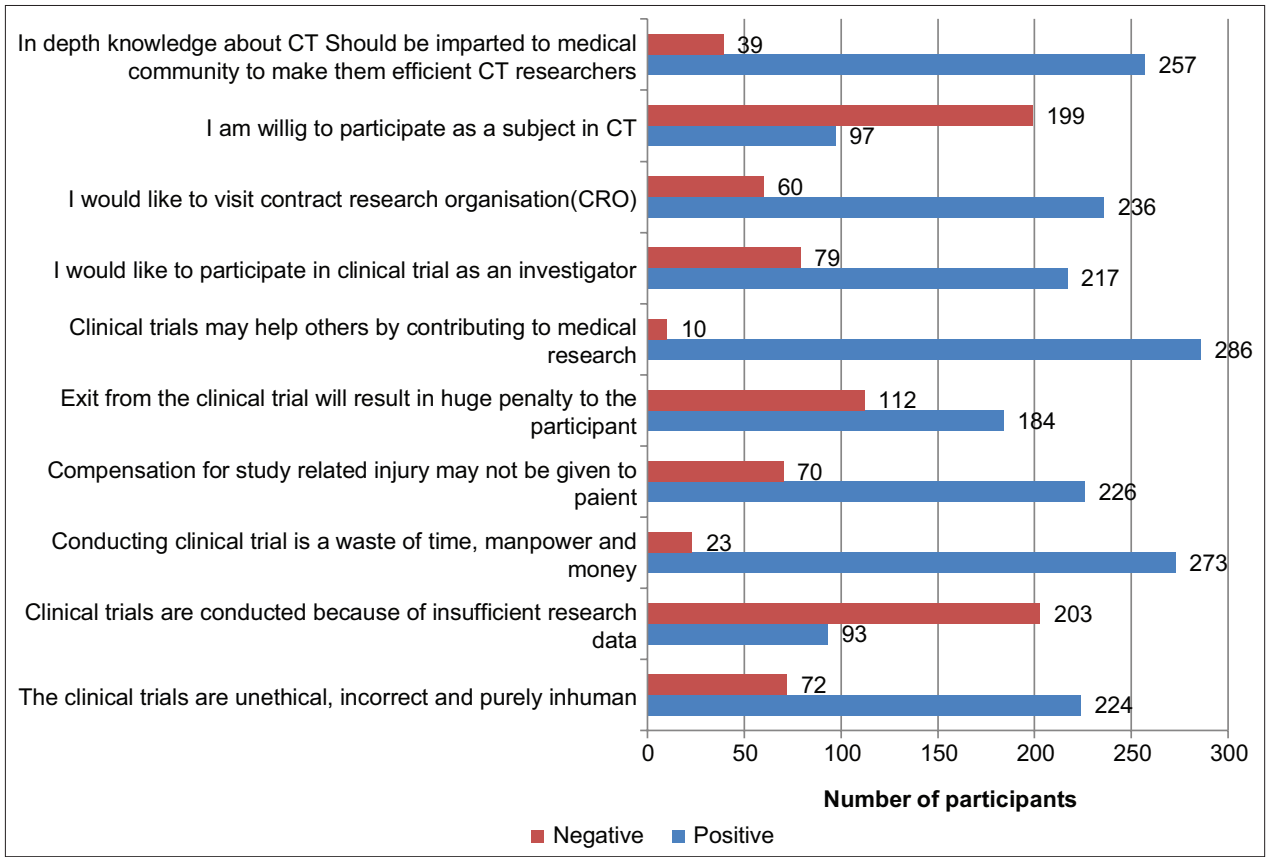

Fig. 1: Attitude of participants about clinical trials

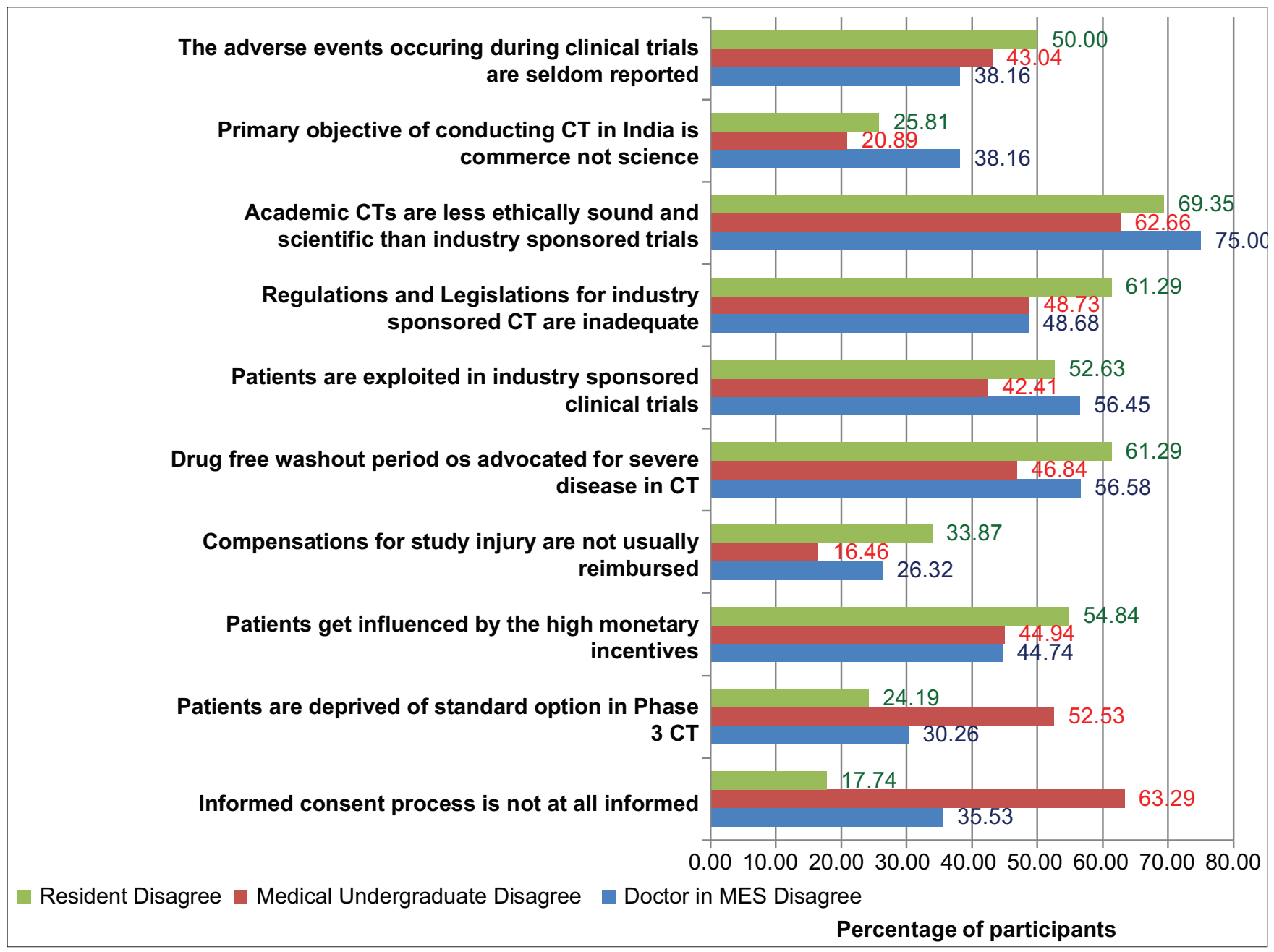

Fig. 2: Perceptions about clinical trials in India

for confidentiality, withdrawal and compensation for study-related injury, and need for registration of trial are lower than those in
Choudhury et al. study group [4]. Medical research knowledge among the undergraduate students is low in India compared to developed 
countries $[13,14]$. The major group of participants $(53.4 \%)$ comprised medical students in this study and that may account for the lower level of knowledge of the participants. However, in contrast to this, Kiruthika et al. stated that the medical undergraduates participating in their study had good awareness about the concepts of CT, Institutional Ethics Committee and average awareness about need of CT, participation in CT, and preclinical and clinical testing. However, their awareness about the trial procedures was poor [15].

Research was not mandatory in Indian undergraduate medical education curriculum so far. However, in other parts of the world, research is an integral part of the curriculum and MBBS students authored many publications of the institutions [13]. Studies have shown that majority of undergraduates were willing to participate in clinical research but only less than a quarter of them reported engagement in research activities [14]. At present, CT is explained with little emphasis in subject of pharmacology and that too without any practical exposure in Indian medical education curriculum [16]. Clinical research project under the guidance of faculty should be made compulsory in the curriculum which would help students to acquire knowledge and experience [17]. The Indian Council of Medical Research organizes short-term studentship to promote aptitude for research among medical undergraduates [18]. Medical Council of India has incorporated clinical research and ethics in the undergraduate teaching curriculum for medical students from the year 2019 [16]. Recent studies have shown lack of knowledge of ethics and Good Clinical Practice guidelines for the proper conduct of CTs among medical undergraduates and investigators $[19,20]$.

The participants in this study had a remarkably positive attitude for CTs with a mean score of $7.07 \pm 1.82$ which is comparable with studies done elsewhere [21]. It is evident from the study that the participants were personally motivated to conduct CTs. Policy-makers at higher level should allocate adequate budget for research and balance the working hours of physicians to permit more research time. There should also be advanced research infrastructure to facilitate data collection and statistical analysis in the institution [22]. Participant recruitment is very important for the success of a trial, and hence, the perception of the participant toward CT is important in recruitment and retention [23]. Even though majority of the participants $(73 \%)$ of this study liked to be the investigator of a CT, only $32 \%$ wished to be recruited in CT as a participant. This shows the negative attitude about participation in CT even among highly educated medical professionals. Chung et al. stated that willingness to participate in CTs is affected by respondent awareness and perception of CTs such as favorable feeling, safety, and necessity of CT. Concern about the adverse effects of the drug was the most prominent barrier and the major reason for unwillingness to participate in a CT. However, they also commented that willingness to participate may not reflect actual enrollment and it is only a behavioral intent [22].

The mean score of the participants on perception about CTs conducted in India is $4.11 \pm 2.02$ which denotes a negative perception. In this study, $38.3 \%$ believed that informed consent was not informed at all while $53.4 \%$ did so in a study by Choudhury et al. [5]. Informed consent is an integral part of CTs. Most patients are afraid of the unknown side effects of novel treatment or they may keep away even from a lifesaving procedure when too many details of adverse reactions are disclosed. This may be one reason why participants in this study had a negative perception about informed consent [24].

In a previous study by Kravitz et al., more than one-third of the participants believed that standard treatment is denied in CT, whereas more than half of the participants did so in our study. Kravitz et al. stated that recruitment remains a challenge for the participants worried about being assigned to arm where they receive less effective treatment [25]. In sharp contrast to Choudhury et al., whose onethird of the participants thought that compensations for study-related injuries are not reimbursed, three-fourth of the participants in this study thought the same. The perceptions that patients are influenced by high monetary incentives, patients are exploited in the industry sponsored CTs and academic CTs, are more scientific and ethical than industry sponsored trials are comparable to the previous study [5]. As industries have a major role in conducting CTs, this misconception has to be resolved for the scientific growth of nation. Ignorance arising from the lack of involvement and adequate sensitization is the cornerstone of overall negative perception toward CTs.

\section{Limitations}

This study was done in a single center. Four questionnaires had to be excluded because they were incomplete. Although we used exclusively closed-ended questions, we recommend use of open-ended questions to further explore the participant's attitude and perception.

\section{CONCLUSION}

The participants had good knowledge and positive attitude about CTs; however, all the three groups of participants had negative perception about CTs currently conducted in India. Sensitizations and opportunities to be a part of CTs will create better perception about CTs in India.

\section{ACKNOWLEDGMENT}

The authors acknowledge the medical undergraduates, junior residents, and staff of the institution who participated in this study.

\section{AUTHORS' CONTRIBUTIONS}

Hari Sankar KN - Protocol preparation, data collection, and manuscript editing. Dhanya Sasidharan Palappallil - Protocol preparation, data collection, data analysis, statistical analysis, manuscript preparation, editing, review, and correspondence. Prabitha Panattil - Protocol preparation, manuscript preparation, editing, and review.

\section{CONFLICTS OF INTEREST}

Nil.

\section{SOURCE OF FUNDING}

Nil.

\section{REFERENCES}

1. Tripathi KD. Pharmacotherapy, clinical pharmacology and drug development. In: Essentials of Medical Pharmacology. $8^{\text {th }}$ ed. New Delhi: Jaypee Brothers Medical Publishers; 2019. p. 84.

2. Gilman G. Drug Invention and the Pharmaceutical Industry. The Pharmacological Basis of Therapeutics. 13 ${ }^{\text {th }}$ ed. New York: McGraw Hill Medical; 2018. p. 6.

3. World Health Organization. Everybody's Business: Strengthening Health Systems to Improve Health Outcomes. WHO's Frame Work for Action. Geneva: World Health Organization; 2007. Available from: https://www.who.int/healthsystems/strategy/everybodys_business. pdf?ua $=1$. [Last accessed on 2020 Jun 06].

4. Odutola AB. Developing countries must invest in access to information for health improvements. J Med Internet Res 2003;5:e5.

5. Choudhury S, Pradhan R, Dubey L, Barman L, Biswas T, Das M, et al. Knowledge and perception regarding clinical trials among doctors of government Medical colleges: A questionnaire-based study. Perspect Clin Res 2016;7:94-9.

6. Choi YJ, Beck SH, Kang WY, Yoo S, Kim SY, Lee JS, et al. Knowledge and perception about clinical research shapes behavior: Face to face survey in Korean general public. J Korean Med Sci 2016;31:674-81.

7. Anderson A, Borfitz D, Getz K. Global public attitudes about clinical research and patient experiences with clinical trials. JAMA Netw Open 2018;1:e182969.

8. Pawar DB, Gawde SR, Marathe PA. Awareness about medical research among resident doctors in a tertiary care hospital: A cross-sectional survey. Perspect Clin Res 2012;3:57-61.

9. Sample Size Calculator; 2020. Available from: https://www. surveysystem.com/sscalc.htm. [Last accessed on 2020 Mar 28].

10. Deodurg PM, Bagewadi HG, Patil BV, Dass AP. Knowledge, attitude and perceptions of $3^{\text {rd }}$ term medical students towards clinical trials in a medical college in southern India. Indian J Pharm Pharmacol 2017;4:125-9.

11. The Report of the Prof Ranjit Roy Chaudhury Expert Committee to 
Formulate Policy and Guidelines for Approval of New Drugs, Clinical Trials and Banning of Drugs; 2020. Available from: http://www.cdsco. nic.in/writereaddata/report_of_dr_ranjit_roy.pdf. [Last accessed on 2020 Mar 28].

12. Rahman S, Majumder MA, Shaban SF, Rahman N, Ahmed M, Bin K, et al. Physician participation in clinical research and trials: Issues and approaches. Adv Med Educ Pract 2011;2:85-93.

13. Kanna RS, Alla J, Krishnakanth K. Knowledge of medical students on clinical trials. Int J Basic Clin Pharmacol 2019;8:1484-8.

14. Abushouk AI, Hatata AN, Omran IM, Youniss MM, Elmansy KF, Meawad AG. Attitudes and perceived barriers among medical students towards clinical research: A cross-sectional study in an Egyptian medical school. J Biomed Educ 2016;2016:5490575.

15. Kiruthika S, Vijayalakshmi S, Geetha K, Parameswari R. A study to assess the knowledge of medical students on clinical trials in Madurai medical college. Int J Sci Res 2016;5:1-3

16. Medical Council of India. Competency Based Undergraduate Curriculum for the Indian Medical Graduate. Vol. 1. India: Medical Council of India; 2018. Available from: https://www.mciindia.org/cms/ wp-content/uploads/2019/01/ug-curriculum-vol-i.pdf. [Last accessed on 2020 Mar 28].

17. Sharma KH, Jindal A. Low awareness of clinical research in India amongst final year medical students and physicians: Need for increased emphasis on clinical research in medical curriculum. Arch Med Health Sci 2014;2:234.

18. Indian Council of Medical Research. Short Term Studentship. India: Indian
Council of Medical Research; 2020. Available from: http://14.139.60.56:84/ about_uspage.aspx. [Last accessed on 2020 Mar 28].

19. Dhodi DK, Thakkar KB, Billa G, Khobragade AA, Sinha SR, Patel SB. Knowledge, attitude and practices of medical students and teachers towards clinical research in a tertiary care hospital in Mumbai-cross sectional survey. J Contemp Med Educ 2013;1:238-44.

20. Vittalrao AM, Kumari KM, Bhat SV, Gill R, Thomson SR. A questionnaire survey on awareness of clinical trials among medical students. Biomed Pharmacol J 2018;11:2005-9.

21. Khalaf AJ, Aljowder AI, Buhamaid MJ, Alansari MF, Jassim GA. Attitudes and barriers towards conducting research amongst primary care physicians in Bahrain: A cross-sectional study. BMC Fam Pract 2019;20:20.

22. Chung SH, Kim EJ, Jeong SH, Park GL. Factors associated with willingness to participate in clinical trials: A nationwide survey study. BMC Public Health 2015;15:10.

23. Kadam RA, Borde SU, Madas SA, Salvi SS, Limaye SS. Challenges in recruitment and retention of clinical trial subjects. Perspect Clin Res 2016;7:137-43.

24. Nijhawan LP, Janodia MD, Muddukrishna BS, Bhat KM, Bairy KL, Udupa N, et al. Informed consent: Issues and challenges. J Adv Pharm Technol Res 2013;4:134-40.

25. Kravitz RL, Paterniti DA, Hay MC, Subramanian S, Dean DE, Weisner T, et al. Marketing therapeutic precision: Potential facilitators and barriers to adoption of n-of- 1 trials. Contemp Clin Trials 2009;30:436-45. 